\title{
COVID-19 and encephalitis
}

\author{
Young-Soo Kim ${ }^{1,2}$ \\ ${ }^{1}$ Department of Neurology, Gyeongsang National University Hospital, Jinju, Korea \\ ${ }^{2}$ Department of Neurology and Institute of Health Science, Gyeongsang National University College of Medicine, Jinju, Korea
}

The current coronavirus disease 2019 (COVID-19) pandemic is caused by severe acute respiratory syndrome coronavirus 2. Due to the increasing number of confirmed cases and accumulating clinical data, in addition to the predominant respiratory symptoms, a significant proportion of patients with COVID-19 experience neurological complications. Presumedly, several mechanisms, such as direct viral effects on the brain parenchyma and endothelium, and activation of the inflammatory and thrombotic pathways, cause these neurological disorders. Herein, the literature focusing on encephalitis among the central nervous system disorders associated with COVID-19 was reviewed.

Keywords: SARS-CoV-2, COVID-19, Encephalitis

\section{Introduction}

Severe acute respiratory syndrome coronavirus 2 (SARS$\mathrm{CoV}-2$ ) infection is characterized by severe respiratory symptoms. In addition to these symptoms, a wide range of central nervous system (CNS) complications in patients with coronavirus disease 2019 (COVID-19) have been reported [1,2]. SARS-CoV-2 infection also has the potential to specifically target the CNS, and some cases of encephalitis have been described in patients admitted to the intensive care unit (ICU) for severe respiratory distress $[3,4]$. The occurrence of encephalitis during SARS-CoV-2 infection independent from acute respiratory distress syndrome (ARDS) has been described in a few case reports, indicating CNS inflammatory-mediated involvement $[5,6]$. However, the neurotropism of SARS-CoV-2 infection and the mechanisms leading to specific brain involvement remain debatable [7-9]. Encephalitis can be defined as inflammation of the brain parenchyma associated with neurologic dysfunction [10]. Patients diagnosed with COVID 19-associated encephalitis have diverse clinical neurological manifestations, including conscious-ness distur- bance, epileptic attacks, and psychotic syndrome. Cerebrospinal fluid (CSF) tests revealed elevated protein, lymphocytes, and cytokines. SARS CoV 2 can be detected in the CSF in certain individuals. Neuroimaging findings include hyperintense signal changes in the white matter and enhancement of meninges on brain magnetic resonance imaging (MRI) [11]. In the present article, several reports of encephalitis associated with SARS-CoV-2 infection were reviewed to summarize the data in the literature and related pathomechanisms.

\section{Main Subjects}

\section{Overview of CNS complications associated with SARS-CoV-2}

In an observational study on CNS manifestations in COVID-19, $31 \%-69 \%$ of patients with severe COVID- 19 and $21 \%$ with nonsevere COVID-19 complained of various CNS symptoms [1,4]. Dizziness (17\%), headache (13\%), impaired consciousness $(8 \%)$, acute stroke $(3 \%)$, ataxia $(<1.0 \%)$, and seizures $(<1.0 \%)$ were the main symptoms reported among hospitalized pa-

\footnotetext{
Received: September 1, 2021 Revised: September 20, 2021 Accepted: September 22, 2021

Correspondence: Young-Soo Kim

Department of Neurology, Gyeongsang National University Hospital, 79 Gangnam-ro, Jinju 52727, Korea

E-mail: youngsookim0127@gmail.com

ORCID: https://orcid.org/0000-0003-1040-372X
}

Copyright (C) 2021 by The Korean Encephalitis and Neuroinflammation Society

This is an open access article distributed under the terms of the Creative Commons Attribution Non-Commercial License (http://creativecommons.org/licenses/by-nc/4.0/) which permits unrestricted non-commercial use, distribution, and reproduction in any medium, provided the original work is properly cited. 
tients [1]. Agitation (69\%), confusion (65\%), signs of corticospinal tract dysfunction (67\%), and impairment of executive function (36\%) were frequent symptoms observed in 64 consecutive patients admitted to two ICUs due to ARDS caused by COVID-19 [4]. In that series, at the time of hospital discharge, $33 \%$ of patients had a dysexecutive syndrome consisting of inattention, disorientation, and poorly organized responses to commands [4].

Retrospective, multicenter, case series studies were conducted in France; based on the analysis of 64 patients with COVID-19 who underwent MRI for neurological symptoms, stroke, leptomeningeal enhancement, and encephalitis were the most common abnormal neuroimaging findings [12]. In particular, encephalitis was present more often in younger patients, and agitation was associated with leptomeningeal enhancement [12]. In the case series reported from England, COVID-19-related neurologic complications were classified into the five categories of encephalopathies, encephalitis, acute disseminated encephalomyelitis (ADEM), myelitis, and ischemic stroke. Among these, the most common was encephalitis, followed by encephalopathies and ischemic stroke [13]. Although differences exist among the reports, 20\%-60\% of patients with COVID-19 had CNS manifestations, and the neurologic symptoms were more frequent in cases of severe respiratory infection. In a retrospective, observational study on neurologic manifestations of 841 patients hospitalized for COVID-19 at two centers in Spain, 57.4\% of patients complained of some form of neurologic symptoms. In particular, disorders of consciousness occurred more frequently in the elderly and severely ill patients [14]. In addition, headache, dizziness, taste and smell dysfunctions, and impaired consciousness were common symptoms in a systematic review of COVID-19 and neurological manifestations, and impaired consciousness was more frequent in severe or critical disease courses [15].

\section{Diagnosis of COVID-19 encephalitis}

Although pathologic examination and testing of brain tissue are considered the "gold standard" diagnostic test for encephalitis syndrome, in the absence of pathologic evidence of brain inflammation, encephalitis has been defined based on selected clinical, laboratory, electroencephalographic, and neuroimaging features. The diagnosis of possible encephalitis requires altered mental status and at least two minor criteria associated with fever, CSF pleocytosis, MRI changes, electroencephalographic changes, new-onset seizures, or focal neurologic deficits [16]. Conversely, encephalopathy refers to a clinical state of altered mental status, manifesting as confusion, disorientation, behavioral changes, or other cognitive impairments, with or without inflammation of brain tissue [16]. Meningitis denotes involvement of the meninges. Clinically, meningitis is characterized by fever, headache, vomiting, and meningeal signs. CSF examination in meningitis usually shows inflammatory changes [17].

According to reports of COVID-19-associated encephalitis, most patients are diagnosed if CNS manifestations are observed, and hyperproteinorrachia and/or pleocytosis observed on CSF examination or MRI are suggestive of encephalitis. In a representative COVID-19-associated encephalitis cohort study, positive CSF reverse transcription (RT) polymerase chain reaction (PCR) results for SARS-CoV-2 were rare $[18,19]$. Standards for encephalitis diagnosis typically are performed by applying the standards for possible encephalitis as previously mentioned. However, consideration of how encephalitis was diagnosed in each study is important.

\section{COVID-19 encephalitis case report}

The first reported case of SARS-CoV-2-related encephalitis was of a 24-year-old Japanese male patient [20]. He was treated with laninamivir (anti-influenza inhaler; Daiichi-Sankyo, Tokyo, Japan) for headache, fatigue, and fever in late February 2020, and his family found him with decreased consciousness on day 9 after symptom onset. A generalized seizure occurred in an ambulance en route to the hospital. Brain computed tomography (CT) showed extensive cerebral edema, and chest CT showed small ground-glass opacities. CSF examination showed a white blood count of $12 / \mu \mathrm{L}$ and a positive SARSCoV-2 PCR test; all other specimens were negative for SARSCoV-2 based on PCR tests. A hyperintense signal change was observed in the right temporal lobe and hippocampus on brain MRI. This was the first encephalitis case associated with COVID-19 for which the patient was admitted for reduced consciousness and seizure and where SARS-CoV-2 RNA was detected in the CSF.

After the aforementioned case report, encephalitis cases associated with COVID-19 continued to be reported from different countries [11]. The initial symptoms varied and included reduced consciousness, seizure attacks, psychotic symptoms, and meningeal irritation signs. Most cases were diagnosed as COVID-19-related encephalitis based on the accompanying neurological symptoms observed during treatment after confirmed SARS-CoV-2 RT-PCR results from nasopharyngeal swab samples. Increased protein and pleocytosis levels were observed on CSF analysis, and $30 \%$ of the patients had posi- 
tive CSF SARS-CoV-2 RT-PCR results. Many case reports did not include brain imaging, possibly due to the difficulty in performing MRI in patients with COVID-19 in many centers at the beginning of the pandemic. Cases with reported MRI results showed subtle, nonspecific encephalitic changes or normal more than specifically for COVID-19-related encephalitis. High-dose steroids or immunoglobulins were administered for treatment after diagnosis, and favorable outcomes were reported for most cases, although death during treatment among elderly patients was reported [11].

\section{COVID-19-associated encephalitis cohort study}

\section{The ENCOVID multicenter study}

The ENCOVID multicenter study conducted in Italy was a landmark cohort study in which the focus was on encephalitis among the various COVID-19-related neurological complications [18]. This was a hospital-based, longitudinal cohort study conducted in 13 neurological units in northern Italy between Lombardy and Veneto, to understand the relationship between COVID-19 and CNS involvement. All patients consecutively admitted to the participating hospitals between February 20, 2020, and May 31, 2020, with symptoms suggestive of encephalitis, were screened for inclusion. Venkatesan's criterion was used as the standard for encephalitis diagnosis [16]. During the study period, 45 patients with encephalitis were recruited, and an association with COVID-19 was observed in 32. Twenty-five cases with MRI, electroencephalography (EEG), and CSF results that could be analyzed with clinical presentation, clinical course, response to treatment, and outcome data were studied. CSF analysis confirmed hyperproteinorrachia and/or pleocytosis in 17 of 25 patients (68.0\%), and positive CSF RT-PCR for SARS-CoV-2 was confirmed in 14 patients but all were negative. ADEM was observed in three patients on MRI, two patients had limbic encephalitis (LE), seven patients had atypical encephalitis, and 13 patients had normal presentation. Patients with imaging patterns for ADEM and LE had slower onset of symptoms and more severe respiratory tract invasion. Furthermore, patients with abnormal MRI findings had poor response to treatment.

\section{Spanish Society of Neurology's COVID-19 Registry}

Another important cohort study was a report from Spanish neurologists regarding neurological involvement in patients with COVID-19 seen between March 17, 2020, and June 6, 2020 [19]. Among various neurological complications, this report was limited to encephalopathy and encephalitis. Neurological symptoms were observed in 232 patients, and patients with encephalopathy or encephalitis accounted for $21.9 \%$ of the study cohort $(n=51)$. In addition, 39 cases of anosmia (16.8\%), 30 of headache (12.9\%), 26 of seizures (11.2\%), 61 of neuromuscular disorder (26.2\%), and 55 of cerebrovascular disease $(23.7 \%)$ were observed. The mean time between onset of infection and onset of neurological symptoms was 8.0 days. Lumbar puncture was performed in $60.8 \%$ of patients, with positive PCR results for SARS-CoV-2 in only one case. Brain MRI studies were performed in $47.0 \%$ of patients and alterations were detected in $7.8 \%$. EEG studies were performed in $41.3 \%$ of cases and alterations were detected in $61.9 \%$. Similar to other reports, the rate of positive SARS-CoV-2 PCR results was very low, and abnormal MRI findings were found in fewer than $10 \%$ of cases. The authors reported that EEG was relatively sensitive and helpful for diagnosis.

5. Mechanisms of COVID-19-associated encephalitis Similar to other well-recognized neuroinvasive human viruses, coronaviruses enter the CNS through three major routes: transsynaptic propagation from the olfactory epithelium, endothelial angiotensin-converting enzyme 2 (ACE2) receptors expressed in brain vessels, or crossing a leaky blood-brain barrier (BBB) affected by systemically produced cytokines [9].

\section{Neuronal pathway}

Some human coronaviruses, including SARS-CoV-1, are considered neurotropic due to their capacity to enter the CNS via the neuronal pathway [21]. Viruses present near the upper respiratory mucosal nerves infect the ends of the sensory and motor nerves and induce retrograde or anterograde transport via kinesins, dynein, and motor proteins [22]. In an intranasal coronavirus infection study in mice, coronaviruses were confirmed to invade the CNS via the olfactory route and gradually reach the brainstem and spinal cord through dispersion [23]. However, as mentioned in the above case series and cohort study, few patients with COVID-19-related encephalitis had positive CSF SARS-CoV-2 PCR results, and we do not believe that neurological involvement via the neuronal pathway is observed in all encephalitis cases.

\section{Blood circulation pathway}

Viruses can enter the CNS without infecting the nerves. The human immunodeficiency virus infects leukocytes and can enter the brain parenchyma via the infected leukocytes. These "Trojan horse mechanisms" are possible because the infected cells can pass through the BBB [24]. In addition, Japanese encephalitis viruses cause increased production of proinflammatory cytokines when they enter the bloodstream, and this increases BBB permeability and accelerates CNS entry [25]. This mechanism is speculated to apply to SARS-COV-2 be- 
cause SARS-COV-2 infections are frequently accompanied by a severe systemic inflammation and an increase in cytokines, and CNS invasion was reportedly positively associated with the severity of respiratory symptoms in most cohort studies [26]. Furthermore, ACE2 receptor expression on the BBB endothelium has been confirmed and can be considered a possible mechanism of CNS entry [6]. However, additional studies are needed to elucidate the mechanism of how SARSCoV-2 enters the CNS.

\section{Immune-mediated injury to the CNS}

SARS-CoV-2 is proposed to cause damage to the CNS by a surge of inflammatory cytokines (mainly interleukin [IL]-6), termed cytokine storm syndrome (CSS), in the same manner as many neurotropic viruses are assumed to induce the production of IL-6 from glial cells, resulting in CSS [27]. In an in vitro study, activated glial cells were observed to cause chronic inflammation and brain damage by producing proinflammatory cytokines such as IL-6, IL-2, IL-5, and tumor necrosis factor- $\alpha$ [28]. SARS-CoV-2 infection of the CNS activates the CD4+ cells of the immune system, which induce macrophages to secrete IL- 6 by producing granulocyte-macrophage colony-stimulating factor. IL-6 is a predominant component of CSS and leads to multiple organ failure, which is a major cause of fatality in COVID-19 [29]. This is supported by reports that treatment with tocilizumab (an IL-6 receptor blocker) resulted in improvement in critically ill COVID-19 patients [30]. Therefore, CSS is a major mechanism used by SARSCoV-2 to indirectly damage the brain. In addition, CSF IL- $1 \beta$, IL-6, and ACE levels were elevated in a case series presenting CSF inflammatory markers in patients with SARS-CoV-2-associated encephalitis. Based on the negative CSF PCR results for SARS-CoV-2 and clinical improvement within three days of symptom onset, the authors estimated the CNS dysfunction in the patient to be a systemic hyperinflammatory response to the virus. They also emphasized that, in this case, anti-IL treatments such as anakinra (targeting IL-1) or tocilizumab and siltuximab (targeting IL-6) would be useful for symptoms of CNS involvement [31].

\section{Conclusion}

Encephalitis, along with cerebrovascular disease and neuromuscular disorder, is a neurological complication often observed in patients with COVID-19. Although the prevalence of encephalitis is difficult to determine based on the available data due to different standards for diagnosis, encephalitis accounts for $20 \%$ of various CNS manifestations. Because posi- tive CSF SARS-COV-2 PCR results are rare in COVID-19-related encephalitis, other mechanisms than the neuronal pathway are likely involved. Responses to immunotherapy are favorable, and the prognosis is good. Brain MRI can show various patterns, such as ADEM and LE; however, in more than half of cases, brain MRI findings appear normal. Therefore, treatment should be decided after considering any neurological symptoms, hyperproteinorrachia and/or pleocytosis found on CSF examination, and abnormal EEG findings. In summary, due to the recent increase in cases, this article review can provide insight into the management of encephalitis in patients with COVID-19.

\section{Conflicts of Interest}

No potential conflict of interest relevant to this article was reported.

\section{References}

1. Mao L, Jin H, Wang M, et al. Neurologic manifestations of hospitalized patients with coronavirus disease 2019 in Wuhan, China. JAMA Neurol 2020;77:683-690.

2. Benussi A, Pilotto A, Premi E, et al. Clinical characteristics and outcomes of inpatients with neurologic disease and COVID-19 in Brescia, Lombardy, Italy. Neurology 2020;95:e910-e920.

3. Dogan L, Kaya D, Sarikaya T, et al. Plasmapheresis treatment in COVID-19-related autoimmune meningoencephalitis: case series. Brain Behav Immun 2020;87:155-158.

4. Helms J, Kremer S, Merdji H, et al. Neurologic features in severe SARSCoV-2 infection. N Engl J Med 2020;382:2268-2270.

5. Pilotto A, Odolini S, Masciocchi S, et al. Steroid-responsive encephalitis in coronavirus disease 2019. Ann Neurol 2020;88:423-427.

6. Bernard-Valnet R, Pizzarotti B, Anichini A, et al. Two patients with acute meningoencephalitis concomitant with SARS-CoV-2 infection. Eur J Neurol 2020;27:e43-e44.

7. Liu K, Pan M, Xiao Z, Xu X. Neurological manifestations of the coronavirus (SARS-CoV-2) pandemic 2019-2020. J Neurol Neurosurg Psychiatry 2020;91:669-670.

8. Leonardi M, Padovani A, McArthur JC. Neurological manifestations associated with COVID-19: a review and a call for action. J Neurol 2020;267:1573-1576

9. Desforges M, Le Coupanec A, Dubeau P, et al. Human coronaviruses and other respiratory viruses: underestimated opportunistic pathogens of the central nervous system? Viruses 2019;12:14.

10. Tunkel AR, Glaser CA, Bloch KC, et al. The management of encephalitis: clinical practice guidelines by the Infectious Diseases Society of 
America. Clin Infect Dis 2008;47:303-327.

11. Lv P, Peng F, Zhang Y, et al. COVID-19-associated meningoencephalitis: a care report and literature review. Exp Ther Med 2021;21:362.

12. Kremer S, Lersy F, Anheim M, et al. Neurologic and neuroimaging findings in patients with COVID-19: a retrospective multicenter study. Neurology 2020;95:e1868-e1882.

13. Paterson RW, Brown RL, Benjamin L, et al. The emerging spectrum of COVID-19 neurology: clinical, radiological and laboratory findings. Brain 2020;143:3104-3120.

14. Chen X, Laurent S, Onur OA, et al. A systematic review of neurological symptoms and complications of COVID-19. J Neurol 2021;268:392402.

15. Romero-Sánchez CM, Díaz-Maroto I, Fernández-Díaz E, et al. Neurologic manifestations in hospitalized patients with COVID-19: the ALBACOVID registry. Neurology 2020;95:e1060-e1070.

16. Venkatesan A, Tunkel AR, Bloch KC, et al. Case definitions, diagnostic algorithms, and priorities in encephalitis: consensus statement of the international encephalitis consortium. Clin Infect Dis 2013;57:11141128.

17. Slooter AJ, Otte WM, Devlin JW, et al. Updated nomenclature of delirium and acute encephalopathy: statement of ten Societies. Intensive Care Med 2020;46:1020-1022.

18. Pilotto A, Masciocchi S, Volonghi I, et al. Clinical presentation and outcomes of severe acute respiratory syndrome coronavirus 2-related encephalitis: the ENCOVID multicenter study. J Infect Dis 2021;223: 28-37.

19. Abenza Abildúa MJ, Atienza S, Carvalho Monteiro G, et al. Encephalopathy and encephalitis during acute SARS-CoV-2 infection. Spanish Society of Neurology COVID-19 Registry. Neurologia (Engl Ed) 2021; 36:127-134.

20. Moriguchi T, Harii N, Goto J, et al. A first case of meningitis/encephalitis associated with SARS-coronavirus-2. Int J Infect Dis 2020;94:55-58.

21. Desforges M, Le Coupanec A, Brison E, Meessen-Pinard M, Talbot PJ.
Neuroinvasive and neurotropic human respiratory coronaviruses: potential neurovirulent agents in humans. Adv Exp Med Biol 2014;807: 75-96.

22. Swanson PA 2nd, McGavern DB. Viral diseases of the central nervous system. Curr Opin Virol 2015;11:44-54.

23. Mori I. Transolfactory neuroinvasion by viruses threatens the human brain. Acta Virol 2015;59:338-349.

24. Koyuncu OO, Hogue IB, Enquist LW. Virus infections in the nervous system. Cell Host Microbe 2013;13:379-393.

25. Unni SK, Růžek D, Chhatbar C, Mishra R, Johri MK, Singh SK. Japanese encephalitis virus: from genome to infectome. Microbes Infect 2011;13:312-321.

26. Channappanavar R, Perlman S. Pathogenic human coronavirus infections: causes and consequences of cytokine storm and immunopathology. Semin Immunopathol 2017;39:529-539.

27. Wan S, Yi Q, Fan S et al. Characteristics of lymphocyte subsets and cytokines in peripheral blood of 123 hospitalized patients with 2019 novel coronavirus pneumonia (NCP). medRxiv 2020 Feb 12 [Epub]. https://doi.org/10.1101/2020.02.10.20021832.

28. Bohmwald K, Gálvez NM, Ríos M, Kalergis AM. Neurologic alterations due to respiratory virus infections. Front Cell Neurosci 2018;12:386.

29. Chen C, Zhang XR, Ju ZY, He WF. Advances in the research of mechanism and related immunotherapy on the cytokine storm induced by coronavirus disease 2019. Zhonghua Shao Shang Za Zhi 2020;36:471475.

30. Zhang C, Wu Z, Li JW, Zhao H, Wang GQ. Cytokine release syndrome in severe COVID-19: interleukin-6 receptor antagonist tocilizumab may be the key to reduce mortality. Int J Antimicrob Agents 2020; 55:105954.

31. Bodro M, Compta Y, Llansó L, et al. Increased CSF levels of IL-1 $\beta$, IL-6, and ACE in SARS-CoV-2-associated encephalitis. Neurol Neuroimmunol Neuroinflamm 2020;7:e821. 